\title{
Equidistribution of holonomy in homology classes for Anosov flows
}

\author{
Richard Sharp ${ }^{1}$
}

Received: 14 June 2017 / Accepted: 24 October 2017 / Published online: 1 December 2017

(C) The Author(s) 2017. This article is an open access publication

\begin{abstract}
We obtain equidistribution results for the holonomies of periodic orbits of Anosov flows lying in a prescribed homology class. We apply this to frame flows.
\end{abstract}

\section{Introduction}

It is well-known that the periodic orbits of hyperbolic flows display considerable asymptotic regularity. For example, there is a uniform asymptotic formula for their counting functions (the so-called Prime Orbit Theorem) and they are equidistributed with respect to the measure of maximal entropy for the flow. Under appropriate conditions, they also exhibit equidistribution in their lifting properties and this is the theme of this paper. More precisely, we will consider the equidistribution of holonomies restricted to periodic orbits in a given homology class.

Without the restriction in homology, this problem was studied by Parry and Pollicott in [11] and Chapter 8 of the monograph [12] for hyperbolic flows. From a different point of view, Sarnak and Wakayama [16] studied the same problem for finite volume quotients of rank 1 locally symmetric spaces and obtained error terms. There was renewed interest in the problem due to the work of Margulis et al. in [9], where there results were extended to geometrically finite quotients. The more delicate problem of equidistribution in a homology was initially studied by Kimoto and Wakayama [8] for finite volume quotients of real hyperbolic space (with an error term). More recently, a paper by Oh and Pan [10] considers the same for convex co-compact quotients of rank 1 locally symmetric spaces as part of an extensive study of mixing properties and horocyclic actions on abelian covers. In this paper, we shall complement this work by taking a more dynamical viewpoint, and consider Anosov flows. Results for geodesic flows and frames flows over compact negatively curved manifolds will then appear as a special case.

Richard Sharp

R.J.Sharp@warwick.ac.uk

1 Mathematics Institute, University of Warwick, Coventry CV4 7AL, UK 
We will now be more precise. Let $G$ be a compact connected Lie group and let $N$ be a principal $G$-bundle over a compact manifold $M$. Let $\psi_{t}: N \rightarrow N$ be a $C^{1}$ flow that commutes with the $G$ action and let $\phi_{t}: M \rightarrow M$ be the quotient flow. Suppose that $\phi_{t}: M \rightarrow M$ is a transitive Anosov flow. (A natural example is where $\phi_{t}$ is the geodesic flow over a compact $d$-dimensional manifold with negative sectional curvatures and $\psi_{t}: N \rightarrow N$ is the corresponding frame flow. In this case, $G=\mathrm{SO}(d-1)$. We will discuss this further in Sect. 7 below.)

Let $\gamma$ be a periodic orbit for $\phi_{t}$. We shall write $l(\gamma)$ for its period. Associated to $\gamma$ we have both its homology class $[\gamma] \in H_{1}(M, \mathbb{Z})$ and its holonomy class $\mathfrak{h}(\gamma)$, which is a conjugacy class in $G$. The latter is defined as follows. Write $\pi: N \rightarrow M$ for the bundle map. Let $x$ be a point on $\gamma$ and let $\tilde{x}$ be a point in $\pi^{-1}(x)$. Then there is a well-defined $g(\tilde{x}) \in G$ such that $\psi_{l(\gamma)}(\tilde{x})=\tilde{x} g(\tilde{x})$. If $\tilde{y}$ is another lift of $x$ then $g(\tilde{y})$ is conjugate to $g(\tilde{x})$ and $\mathfrak{h}(\gamma)$ is conjugacy class this defines.

Let $\mathcal{P}(\phi)$ denote the set of prime periodic orbits of $\phi_{t}: M \rightarrow M$ and let $\mathcal{P}_{T}(\phi)=\{\gamma \in$ $\mathcal{P}(\phi): l(\gamma) \leq T\}$. If $\phi$ is topologically weak-mixing then it is well-known that

$$
\# \mathcal{P}_{T}(\phi) \sim \frac{e^{h T}}{h T}, \quad \text { as } T \rightarrow \infty,
$$

where $\sim$ means that the ratio of the two sides converges to one.

We will say that $\phi_{t}: M \rightarrow M$ is homologically full if the map from $\mathcal{P}(\phi)$ to $H_{1}(M, \mathbb{Z})$ defined by $\gamma \mapsto[\gamma]$ is a surjection. For $\alpha \in H_{1}(M, \mathbb{Z})$, write $\mathcal{P}_{T}(\phi, \alpha)=\{\gamma \in$ $\left.\mathcal{P}_{T}(\phi):[\gamma]=\alpha\right\}$. If $\phi$ is homologically full then, for each $\alpha, \# \mathcal{P}_{T}(\phi, \alpha)$ grows exponentially fast (with polynomial correction depending on the first Betti number of $M$ ) but the growth rate

$$
h^{*}:=\lim _{T \rightarrow \infty} \frac{1}{T} \log \# \mathcal{P}_{T}(\phi, \alpha)>0
$$

(which is independent of $\alpha$ ) may be strictly smaller than $h$. For a more precise statement, see Proposition 3.1 below.

We are interested in the distribution of the holonomies $\mathfrak{h}(\gamma)$ for $\gamma$ in a fixed homology class. Under natural hypotheses, we shall show that they are equidistributed with respect to the (normalised) Haar measure on $G$. We say that $F: G \rightarrow \mathbb{C}$ is a class function if it is constant on each conjugacy class.

Definition 1.1 Let $\psi_{t}: N \rightarrow N$ and $\phi_{t}: M \rightarrow M$ be as above. We say that $\phi_{t}$ has homologyequidistributed holonomy if, for each $\alpha \in H_{1}(M, \mathbb{Z})$ and continuous class function $F: G \rightarrow$ $\mathbb{C}$,

$$
\frac{1}{\# \mathcal{P}_{T}(\phi, \alpha)} \sum_{\gamma \in \mathcal{P}_{T}(\phi, \alpha)} F(\mathfrak{h}(\gamma)) \rightarrow \int F d \text { Haar, } \quad \text { as } T \rightarrow \infty \text {. }
$$

Our main results are the following. The first deals with the case where $G$ is semisimple and, in addition to the assumptions on $\phi_{t}$, requires only that $\psi_{t}$ is transitive.

Theorem 1.2 Let $G$ be a compact connected semisimple Lie group. Let $N$ be a principal $G$-bundle over $M$ and let $\psi_{t}: N \rightarrow N$ be a $C^{1}$ flow that commutes with the $G$-action. Let $\phi_{t}: M \rightarrow M$ be the quotient flow. Assume that

(i) $\psi_{t}$ is transitive;

(ii) $\phi_{t}$ is a homologically full transitive Anosov flow. 
Then $\phi_{t}$ has homology-equidistributed holonomy.

The next result holds when $G$ is no longer assumed to be semisimple but requires an additional a priori condition on the distribution of holonomy of null-homologous periodic orbits.

Theorem 1.3 Let $G$ be a compact connected Lie group. Let $N$ be a principal G-bundle over $M$ and let $\psi_{t}: N \rightarrow N$ be a $C^{1}$ flow that commutes with the $G$-action. Let $\phi_{t}: M \rightarrow M$ be the quotient flow. Assume that

(i) $\psi_{t}$ is transitive;

(ii) $\phi_{t}$ is a homologically full transitive Anosov flow;

(iii) we have

$$
e^{i a l(\gamma)} \chi(\mathfrak{h}(\gamma))=1 \quad \forall \gamma \in \mathcal{P}(\phi, 0),
$$

for $a \in \mathbb{R}$ and $\chi: G \rightarrow \mathbb{T}^{1}$ a one-dimensional representation of $G$, only when $a=0$ and $\chi$ is trivial.

Then $\phi_{t}$ has homology-equidistributed holonomy.

We say that $\phi_{t}: M \rightarrow M$ has time-reversal symmetry if there is a fixed point free involution $\iota: M \rightarrow M$ such that $\phi_{t} \circ \iota=\iota \circ \phi_{-t}$. This holds for geodesics flows, where $M=S V$ is the unit-tangent bundle over a manifold $V$ and $\iota$ is the map that reverses unit tangent vectors: $\iota(x, v)=(x,-v)$. In this case, the conclusion of Theorem 1.3 holds subject to a simpler hypothesis on the holonomies.

Theorem 1.4 If $\phi_{t}: M \rightarrow M$ has time-reversal symmetry then Theorem 1.3 still holds if we replace (iii) by the condition that we have

$$
\chi(\mathfrak{h}(\gamma))=1 \quad \forall \gamma \in \mathcal{P}(\phi, 0),
$$

for $\chi: G \rightarrow \mathbb{T}^{1}$ a one-dimensional representation of $G$, only when $\chi$ is trivial.

Remark 1.5 The above theorems still hold if, instead of homology, we consider any regular abelian cover of $M$ and ask about the holonomies of periodic orbits in a given Frobenius class for the cover. In this case, $b$ is replaced by the rank of the cover.

We now briefly outline the contents of the paper. In Sect. 2, we recall some material about Lie groups and representations that will be needed later. In Sect. 3, we discuss Anosov flows in greater detail and introduce the $L$-functions that we shall use to prove our results. In Sect. 4 , we introduce symbolic dynamical systems as a tool for analysing the $L$-functions. In Sect. 5 , we proof the equidistribution results srtated about and in Sect. 6 we give a brief discussion of the special case of frame flows.

\section{Lie groups and representations}

We will need a small amount of theory relating to compact Lie groups and their representations. Let $G$ be a compact connected Lie group. We shall be interested in its space of irreducible unitary representations. More precisely, let $\mathrm{U}(d)$ denote the group of $d \times d$ unitary matrices. Then a $d$-dimensional unitary representation is a continuous homomorphism $R: G \rightarrow \mathrm{U}(d)$. The character $\chi$ of $R$ is its trace $\chi(g)=\operatorname{Trace}(R(g))$ and we write $R=R_{\chi}$. 
If $R_{\chi}$ is one-dimensional, in which case $\mathrm{U}(1)=\mathbb{T}^{1}$, then it is equal to its character. (Here and throughout, we use the notation $\mathbb{T}^{k}=\mathbb{R}^{k} / \mathbb{Z}^{k}$.) A $d$-dimensional representation $R_{\chi}$ is irreducible if $\mathbb{C}^{d}$ has no $R_{\chi}(G)$-invariant closed subspaces, apart from $\{0\}$ and $\mathbb{C}^{d}$ itself. Two such representations $R$ and $R^{\prime}$ are isomorphic if $R^{\prime}(g)=U^{-1} R(g) U$, for all $g \in G$, for some unitary matrix $U$, in which case they have the same character.

We say that a continuous function $F: G \rightarrow \mathbb{C}$ is a class function if it is constant on conjugacy classes. Let $\mathcal{C}(G)$ denote the set of continuous class functions on $G$. The following is part of the Peter-Weyl theorem.

Proposition 2.1 The set of finite linear combinations of characters of irreducible unitary representations of $G$ is uniformly dense in $\mathcal{C}(G)$.

The structure of compact connected Lie groups may be described by the following result. Recall that a Lie group is simple if it has no non-trivial proper closed normal subgroups. Also that the centre of a group $G$ is the set $\{h \in G: h g=g h \forall g \in G\}$ and that this is a normal subgroup. Any connected component of the centre in a torus.

Proposition 2.2 [15, Theorem 6.4.2] Let $G$ be a compact connected Lie group. Then $G$ is isomorphic to

$$
\left(\mathbb{T}^{m} \times G_{1} \times \cdots \times G_{m}\right) / Z,
$$

where $\mathbb{T}^{m}$ is the connected component of the centre of $G$, the factors $G_{1}, \ldots, G_{n}$ are simple Lie groups, and $Z$ is a finite subgroup of the centre of the product. Furthermore, this expression is unique up to the order of the simple factors.

A Lie group is called semisimple if its Lie algebra is semisimple (i.e. if the Lie algebra is a direct sum of simple Lie algebras). In our situations, a compact connected Lie group $G$ is semisimple if and only if $m=0$ in the decomposition in Proposition 2.2, and this is equivalent to $G$ having finite centre.

Lemma 2.3 Let $G$ be a compact connected Lie group. Then $G$ is semisimple if and only if $G$ has no non-trivial one-dimensional unitary representations.

Proof Consider the decomposition of $G$ in Propostion 2.2. If $m=0$ this reduces to $\left(G_{1} \times\right.$ $\left.\cdots \times G_{m}\right) / Z$. It is an easy fact that since each $G_{i}$ is a simple Lie group, we have $G_{i}=$ $\left[G_{i}, G_{i}\right]$, which is to say $G_{i}$ has no non-trivial characters. Clearly, this extend to the product $G_{1} \times \cdots \times G_{n}$. However, if $\chi:\left(G_{1} \times \cdots \times G_{n}\right) / Z \rightarrow \mathbb{T}^{1}$ is a non-trivial character then it lifts to a non-trivial character on $G_{1} \times \cdots \times G_{n}$. On the other hand, if $m \geq 1$ then $G$ has a non-trivial character.

\section{Anosov flows and $L$-functions}

We begin by discussing some generalities about flows. A continuous flow $\rho_{t}: X \rightarrow X$ on a compact metric space $X$ is called transitive if it has a dense orbit and is called topologically weak-mixing if the only continuous solution $f: X \rightarrow \mathbb{C}$ to $f\left(\rho_{t} x\right)=e^{i a t} f(x)$ is given by $a=0$ and $f$ constant.

We now consider a situation with more structure. Let $G$ be a compact connected Lie group. and let $N$ be a principal $G$-bundle over a smooth compact Riemannian manifold $M$. (This means that $N$ is a fibre bundle over $M$ equipped with a smooth action $N \times G \rightarrow N$ which 
preserves and acts freely and transitively on each fibre. Furthermore, we can identify $M$ with the quotient space $N / G$.) Suppose that $\psi_{t}: N \rightarrow N$ is a $C^{1}$ flow on $N$ which commutes with the $G$-action. Then $\psi_{t}$ induces a well-defined flow $\phi_{t}: M \rightarrow M$. We assume that $\psi_{t}$ is transitive; this implies that $\phi_{t}$ is transitive. In addition, we will assume that $\phi_{t}$ is an Anosov flow.

A $C^{1}$ flow $\phi_{t}: M \rightarrow M$ on a smooth compact Riemannian manifold $M$ is called Anosov if there is a continuous $D \phi$-invariant splitting $T M=E^{0} \oplus E^{s} \oplus E^{u}$, where $E^{0}$ is the line bundle generated by the flow and where there exist constants $C>0$ and $\lambda>0$ such that

(i) $\left\|D \phi_{t}(v)\right\| \leq C e^{-\lambda t}\|v\|$, for all $v \in E^{s}$ and $t \geq 0$; and

(ii) $\left\|D \phi_{-t}(v)\right\| \leq C e^{-\lambda t}\|v\|$, for all $v \in E^{u}$ and $t \geq 0$.

We will use $\gamma$ to denote a typical periodic $\phi$-orbit and write $l(\gamma)$ for its period. As in the introduction, let $\mathcal{P}(\phi)$ denote the set of prime periodic $\phi$-orbits, where a periodic orbit $\gamma=\left\{\phi_{t}(x): 0 \leq t \leq l(\gamma)\right\}$ is prime if $\phi_{t}(x) \neq x$ for all $0<t<l(\gamma)$.

We will be interested in the first homology groups, $H_{1}(M, \mathbb{Z})$ and $H_{1}(M, \mathbb{R})=$ $H_{1}(M, \mathbb{Z}) \otimes_{\mathbb{Z}} \mathbb{R} . H_{1}(M, \mathbb{Z})$ is isomorphic to $\mathbb{Z}^{b} \times A$, where $b \geq 0$ is the first Betti number of $M$ and where $A$ is a finite abelian group. Each $\gamma \in \mathcal{P}(\phi)$ defines a homology class $[\gamma] \in H_{1}(M, \mathbb{Z})$ and we write $[\gamma]_{\mathrm{TF}} \in \mathbb{Z}^{b}$ for its torsion-free part. We say that $\phi_{t}$ is homologically full if the map $[\cdot]: \mathcal{P}(\phi) \rightarrow H_{1}(M, \mathbb{Z})$ is a surjection.

An Anosov flow fails to be topologically weak-mixing if and only if it is the constant suspension of a diffeomorphism [13]. As a consequence, if $\phi_{t}$ is homologically full, then it is automatically topologically weak-mixing (since, for example, a suspension flow has no null homologous periodic orbits) [17].

As above, let $\mathcal{P}_{T}(\phi)=\{\gamma \in \mathcal{P}(\phi): l(\gamma) \leq T\}$ and, for $\alpha \in H_{1}(M, \mathbb{Z})$, let $\mathcal{P}_{T}(\phi, \alpha)=$ $\left\{\gamma \in \mathcal{P}_{T}(\phi):[\gamma]=\alpha\right\}$. The following was proved in [17]. (More precise results with error terms appear in [14].)

Proposition 3.1 Let $\phi_{t}: M \rightarrow M$ be a homologically full transitive Anosov flow. Then there exists $0<h^{*} \leq h, \xi \in H^{1}(M, \mathbb{R})$ and a constant $C>0$ such that, for each $\alpha \in H_{1}(M, \mathbb{Z})$,

$$
\# \mathcal{P}_{T}(\phi, \alpha) \sim C e^{-\left\langle\xi, \alpha_{\mathrm{TF}}\right\rangle} \frac{e^{h^{*} T}}{T^{1+b / 2}}, \quad \text { as } T \rightarrow \infty,
$$

where $\alpha_{\mathrm{TF}}$ is the torsion-free part of $\alpha$.

The quantities $h^{*}$ and $\xi$ appearing in the asymptotic may be characterised in the following ways. For $F \in C(M, \mathbb{R})$ we define its pressure $P(F)$ by

$$
P(F)=\sup \left(h_{\phi}(m)+\int F d m\right)
$$

where the supremum is taken over $\phi$-invariant probability measures $m$ with entropy $h_{\phi}(m)$. Let $\omega$ be a closed 1-form on $M$, giving rise to a (de Rham) cohomology class $[\omega] \in H^{1}(M, \mathbb{R})$, and let $Z: M \rightarrow T M$ be the vector field generating the flow. Define $\beta: H^{1}(M, \mathbb{R}) \rightarrow \mathbb{R}$ by $\beta([\omega])=P(\omega(Z))$. Then $\beta$ is strictly convex and has a finite minimum occurring at $\xi \in$ $H^{1}(M, \mathbb{R})$. If $m$ is a $\phi$-invariant measure then we define its asymptotic cycle $\Phi_{m} \in H_{1}(M, \mathbb{R})$ by the duality $\Phi_{m}([\omega])=\int \omega(Z) d m$. Then we have $h^{*}=\beta(\xi)=\sup \left\{h_{\phi}(m): \Phi_{m}=0\right\}$. See [17] for further details.

Proposition 3.1 was proved by considering $L$-functions indexed by the character group of $H_{1}(M, \mathbb{Z})$, i.e. $\mathbb{T}^{b} \times \widehat{A}$. In order to study the holonomy induced by $\psi_{t}$, we shall consider augmented $L$-functions which take into account the representations of $G$. We will follow 
the approach of [11] and Chapter 8 of [12]. To simplify the exposition, we shall assume that $A$ is trivial, so our character group for homology will just be $\mathbb{T}^{b}$ and $[\gamma]_{\mathrm{TF}}=[\gamma]$. Given the results of [17], the presence of torsion creates no extra difficulties. Let $R_{\chi}$ be an $m$-dimensional $(m \geq 1)$ irreducible unitary representation of $G$ with character $\chi$. We will consider the $L$-function

$$
L(s, t, \chi)=\prod_{\gamma \in \mathcal{P}(\phi)}\left(\operatorname{det}\left(I-R_{\chi}(\mathfrak{h}(\gamma)) e^{-s l(\gamma)+2 \pi i\langle t,[\gamma]\rangle+\langle\xi,[\gamma]\rangle}\right)\right)^{-1},
$$

where $t \in \mathbb{T}^{b}$. We will analyse these functions in Sects. 5 and 6 .

\section{Shifts of finite type}

In this section we introduce the symbolic dynamical systems, shifts of finite type, which may be used to model Anosov flows. We begin with some definitions. For $k \geq 2$, let $A$ be a $k \times k$ matrix with entries 0 and 1 . We define the two-sided shift of finite type associated to $A$ to be the space

$$
\Sigma=\left\{x=\left(x_{i}\right)_{i=-\infty}^{\infty} \in\{1, \ldots, k\}^{\mathbb{Z}}: A\left(x_{i}, x_{i+1}\right)=1 \forall i \in \mathbb{Z}\right\},
$$

equipped with the shift map $\sigma: \Sigma \rightarrow \Sigma$ defined by $(\sigma x)_{i}=x_{i+1}$. Similarly, we define the one-sided shift of finite type to be the space

$$
\Sigma^{+}=\left\{x=\left(x_{i}\right)_{i=0}^{\infty} \in\{1, \ldots, k\}^{\mathbb{Z}^{+}}: A\left(x_{i}, x_{i+1}\right)=1 \forall i \in \mathbb{Z}^{+}\right\},
$$

equipped with the shift map $\sigma: \Sigma^{+} \rightarrow \Sigma^{+}$defined by $(\sigma x)_{i}=x_{i+1}$. We note that there is an obvious correspondence between the periodic orbits for $\sigma: \Sigma \rightarrow \Sigma$ and $\sigma: \Sigma^{+} \rightarrow \Sigma^{+}$.

Define an equivalence relation $\sim_{n}$ on $\Sigma$ by $x \sim_{n} y$ if $x_{i}=y_{i}$ for $|i| \leq n$ and, for $0<\theta<1$, define a metric $d_{\theta}$ by $d_{\theta}(x, y)=\theta^{n}$ if $x \sim_{n} y$ but $x \nsim_{n+1} y$. This makes $\Sigma$ into a compact metric space. For a metric space $Y$, let $F_{\theta}(\Sigma, Y)$ denote the space of $d_{\theta}$-Lipschitz functions $f: \Sigma \rightarrow Y$. We will be particularly interested in the cases $Y=\mathbb{R}$ or $\mathbb{C}$, or, more generally, $\mathbb{C}^{m}, m \geq 1$. These are Banach spaces with respect to the norm $\|\cdot\|_{\theta}:=\|\cdot\|_{\infty}+|\cdot|_{\theta}$, where $|f|_{\theta}$ is the best choice of Lipschitz constant for $f$. We make similar definitions on $\Sigma^{+}$, except that, when defining $\sim_{n}$, the condition $|i| \leq n$ is replaced by $0 \leq i \leq n$.

We say that two functions $f, g \in F_{\theta}(\Sigma, \mathbb{C})$ are cohomologous if there is a continuous function $u: \Sigma \rightarrow \mathbb{C}$ such that $f=g+u \circ \sigma-u$. Write $f^{n}(x)=f(x)+f(\sigma x)+\cdots+$ $f\left(\sigma^{n-1} x\right)$. If $f$ and $g$ are cohomologous then $f^{n}(x)=g^{n}(x)$ whenever $\sigma^{n} x=x$. We also say that two functions $\kappa, \lambda \in F_{\theta}(\Sigma, G)$ are cohomologous if there exists $\zeta \in C(\Sigma, G)$ such that $\kappa=(\zeta \circ \sigma)^{-1} \lambda \zeta$. Write $\kappa_{n}(x)=\kappa\left(\sigma^{n-1} x\right) \cdots \kappa(\sigma x) \kappa(x)$. If $\kappa$ and $\lambda$ are cohomologous then $\kappa_{n}(x)$ and $\lambda_{n}(x)$ are conjugate in $G$ whenever $\sigma^{n} x=x$. Again, the same holds with $\Sigma$ replaced by $\Sigma^{+}$.

We define the pressure $P(f)$ of a function $f \in C(\Sigma, \mathbb{R})$ or $C\left(\Sigma^{+}, \mathbb{R}\right)$ by

$$
P(f)=\sup \left(h_{\sigma}(v)+\int f d v\right),
$$

where the supremum is taken over $\sigma$-invariant probability measures on $\Sigma$ or $\Sigma^{+}$. It is also characterised by the limit

$$
P(f)=\lim _{n \rightarrow \infty} \frac{1}{n} \log \sum_{\sigma^{n} x=x} e^{f^{n}(x)} .
$$


For $f \in F_{\theta}\left(\Sigma^{+}, \mathbb{C}\right)$, we define the transfer operator $\mathcal{L}_{f}: F_{\theta}\left(\Sigma^{+}, \mathbb{C}\right) \rightarrow F_{\theta}\left(\Sigma^{+}, \mathbb{C}\right)$ by

$$
\left(\mathcal{L}_{f} w\right)(x)=\sum_{\sigma y=x} e^{f(y)} w(y)
$$

If $f$ is real-valued then the spectral radius of $\mathcal{L}_{f}$ is equal to $e^{P(f)}$ and, moreover, $e^{P(f)}$ is a simple eigenvalue and the rest of the spectrum is contained in a disc of strictly smaller radius. More generally, if $\operatorname{Im} f$ is cohomologous to a constant $a \in \mathbb{R}$, then $e^{P(\operatorname{Re} f)+i a}$ is a simple eigenvalue for $\mathcal{L}_{f}$ and the rest of the spectrum is contained in a disc of strictly smaller radius, in which case, we define $e^{P(f)}=e^{P(\operatorname{Re} f)+i a}$. We may then use eigenvalue perturbation theory to extend $f \mapsto e^{P(f)}$ as an analytic function on a larger domain $\mathcal{D} \subset F_{\theta}\left(\Sigma^{+}, \mathbb{C}\right)$.

The definition of transfer operator may be extended to include twisting by unitary matrices. For a function $U \in F_{\theta}\left(\Sigma^{+}, \mathrm{U}(m)\right), m \geq 1$, we define the twisted transfer operator $\mathcal{L}_{f, U}$ : $F_{\theta}\left(\Sigma^{+}, \mathbb{C}^{m}\right) \rightarrow F_{\theta}\left(\Sigma^{+}, \mathbb{C}^{m}\right)$ by

$$
\left(\mathcal{L}_{f, U}\right) w(x)=\sum_{\sigma y=x} e^{f(y)} U(y) w(y) .
$$

We will discuss these operators further in the next section.

\section{Symbolic dynamics for Anosov flows}

The Anosov flow $\phi_{t}: M \rightarrow M$ may be modelled by a suspension flow over $\Sigma$ and this in turn may be transferred to a suspension semiflow over $\sigma: \Sigma^{+} \rightarrow \Sigma^{+}$. More precisely, we have the following [1]. Given a strictly positive function $r \in F_{\theta}(\Sigma, \mathbb{R})$, we define

$$
\Sigma^{r}=\{(x, s) \in \Sigma \times \mathbb{R}: 0 \leq s \leq r(x)\} / \sim,
$$

where $(x, r(x)) \sim(\sigma x, 0)$, and the suspension flow $\sigma_{t}^{r}: \Sigma^{r} \rightarrow \Sigma^{r}$ by $\sigma_{t}^{r}(x, s+t)$ modulo the identifications. We note that there is an obvious correspondence between the periodic orbits of $\sigma_{t}^{r}$ and $\sigma$ and that if a periodic $\sigma^{r}$-orbit corresponds to $\sigma^{n} x=x$ then this orbit has period $r^{n}(x)$.

Given $\phi_{t}$, we can find a mixing shift of finite type $\sigma: \Sigma \rightarrow \Sigma$ and strictly positive function $r \in F_{\theta}\left(\Sigma^{+}, \mathbb{R}\right.$ ) (for some choice of $0<\theta<1$ ) so that there is a surjective Hölder continuous semi-conjugacy $\pi: \Sigma^{r} \rightarrow M$ between $\sigma_{t}^{r}$ and $\phi_{t}$ that is one-to-one on a residual set. This induces a surjection from the set of prime $\sigma$-periodic orbits to $\mathcal{P}(\phi)$. In addition to $r$, we can choose a locally constant function $f: \Sigma \rightarrow \mathbb{Z}^{b}$ (which we may arrange to only depend on two co-ordinates, $\left.f(x)=f\left(x_{0}, x_{1}\right)\right)$ and a function $\kappa \in F_{\theta}\left(\Sigma^{+}, G\right)$ such that, if $\left\{x, \sigma x, \ldots, \sigma^{n-1} x\right\}$ (with $\sigma^{n} x=x$ ) is mapped to $\gamma$ then

(i) $r^{n}(x)=l(\gamma)$;

(ii) $f^{n}(x)=[\gamma]$;

(iii) $\kappa_{n}(x)$ is in the conjugacy class $\mathfrak{h}(\gamma)$.

We now pass to the one-sided shift $\sigma: \Sigma^{+} \rightarrow \Sigma^{+}$. The function $f$ is clearly defined as a (locally constant) function $f: \Sigma^{+} \rightarrow \mathbb{Z}^{b}$. Furthermore, we may choose a function $r^{\prime} \in F_{\theta^{1 / 2}}(\Sigma, \mathbb{R})$ that is cohomologous to $r$ and which only depends on the future coordinates, i.e. $r^{\prime}\left(\left(x_{i}\right)_{i=-\infty}^{\infty}\right)=r^{\prime}\left(\left(x_{i}\right)_{i=0}^{\infty}\right)$ ([12], Proposition 1.2). Thus we may regard $r^{\prime}$ as an element of $F_{\theta^{1 / 2}}\left(\Sigma^{+}, \mathbb{R}\right)$ and, when $\sigma^{n} x=x$, we have $\left(r^{\prime}\right)^{n}(x)=r^{n}(x)$ (where we are using the obvious correspondence between periodic points of the one-sided and two-sided shifts). Also, there exists $\kappa^{\prime} \in F_{\theta^{1 / 2}}(\Sigma, G)$ that is cohomologous to $\kappa$ and which only depends on the future 
coordinates ([12], Appendix II). Although this cohomology does not give equality of $\kappa_{n}^{\prime}(x)$ and $\kappa_{n}(x)$, for $\sigma^{n} x=x$, if $R_{\chi}$ is a unitary representation of $G$ with character $\chi$ then

$$
\chi\left(\kappa_{n}^{\prime}(x)\right)=\operatorname{Trace}\left(R_{\chi}\left(\kappa_{n}^{\prime}(x)\right)\right)=\operatorname{Trace}\left(R_{\chi}\left(\kappa_{n}(x)\right)\right)=\chi\left(\kappa_{n}(x)\right),
$$

whenever $\sigma^{n} x=x$. From now on, we will suppress the primes and the square root, and suppose that $r \in F_{\theta}\left(\Sigma^{+}, \mathbb{R}\right)$ and $\kappa \in F_{\theta}\left(\Sigma^{+}, G\right)$.

We note that $\kappa: \Sigma^{+} \rightarrow G$ induces a skew-product system $\tilde{\sigma}: \Sigma^{+} \times G \rightarrow \Sigma^{+} \times G$ defined by $\tilde{\sigma}(x, g)=(\sigma x, \kappa(x) g)$. The next lemma follows immediately from the transitivity of $\psi_{t}: N \rightarrow N$.

Lemma $5.1 \tilde{\sigma}: \Sigma^{+} \times G \rightarrow \Sigma^{+} \times G$ is transitive.

There correspondence between periodic $\sigma$-orbits and periodic $\phi$-orbits is not a bijection. However, the discrepancy caused by the overcounting has small growth relative to the leading asymptotics [1]. In fact, it follows from the analysis of [17] that, if we define a symbolic $L$-function

$$
L_{\sigma}(s, t, \chi)=\exp \sum_{n=1}^{\infty} \frac{1}{n} \sum_{\sigma^{n} x=x} \chi\left(\kappa_{n}(x)\right) e^{-s r^{n}(x)+\left\langle\xi+2 \pi i t, \psi^{n}(x)\right\rangle},
$$

then $L(s, t, \chi) / L_{\sigma}(s, t, \chi)$ is non-zero and analytic for $\operatorname{Re}(s)>h^{*}-\epsilon$. Thus, to get the results we require, it will be sufficient to study the functions $L_{\sigma}(s, t, \chi)$.

Since

$$
\left|\sum_{\sigma^{n} x=x} \chi\left(\kappa_{n}(x)\right) e^{-s r^{n}(x)+\left\langle\xi+2 \pi i t, f^{n}(x)\right\rangle}\right| \leq m \sum_{\sigma^{n} x=x} e^{-\operatorname{Re}(s) r^{n}(x)+\left\langle\xi, f^{n}(x)\right\rangle},
$$

we have that $L_{\sigma}(s, t, \chi)$ converges to a non-zero analytic function for $P(-\operatorname{Re}(s) r+\langle\xi, f\rangle)<$ 0 , i.e. for $\operatorname{Re}(s)>h^{*}$. In order to make progress, we need to extend it to a larger domain and this in turn depends on the spectrum of twisted transfer operators.

Let $R_{\chi}$ be an $m$-dimensional irreducible representation of $G$ and write $U=R_{\chi} \circ \kappa$ : $\Sigma^{+} \rightarrow \mathrm{U}(m)$. The following is Theorem 8.1, Theorem 8.3 and Proposition 8.3 of [12] (cf. also Proposition 3.7 of [10]).

Lemma 5.2 Let $f=u+i v \in F_{\theta}\left(\Sigma^{+}, \mathbb{C}\right)$ and let $\rho\left(\mathcal{L}_{f, U}\right)$ denote the spectral radius of $\mathcal{L}_{f, U}: F_{\theta}\left(\Sigma^{+}, \mathbb{C}^{m}\right) \rightarrow F_{\theta}\left(\Sigma^{+}, \mathbb{C}^{m}\right)$.

(i) We have $\rho\left(\mathcal{L}_{f, U}\right) \leq e^{P(u)}$. Furthermore, if $\rho\left(\mathcal{L}_{f, U}\right)=e^{P(u)}$ then there exists $w \in$ $F_{\theta}\left(\Sigma^{+}, \mathbb{C}^{m}\right), w \neq 0$, and $a \in \mathbb{R}$ such that

$$
\mathcal{L}_{f, U} w=e^{P(u)+i a} w .
$$

(ii) If $m>1$ then $\rho\left(\mathcal{L}_{f, U}\right)<e^{P(u)}$.

Proof Part (i) is proved using adaptations of the theory developed earlier in [12]. It may be useful to give a proof of part (ii). Without loss of generality, we may assume that $P(u)=0$. Suppose $\rho\left(\mathcal{L}_{f, U}\right)=1$. By (i), there exists $w \in F_{\theta}\left(\Sigma^{+}, \mathbb{C}^{m}\right), w \neq 0$, and $a \in \mathbb{R}$ such that

$$
\mathcal{L}_{f, U} w=e^{i a} w .
$$

Using a standard convexity argument (as in [12]), it follows that

$$
e^{i v(y)} U(y) w(y)=e^{i a} w(\sigma y)
$$


for all $y \in \Sigma^{+}$. Rewriting, we have

$$
e^{i v(y)-i a} w(y)=U(y)^{-1} w(\sigma y) .
$$

for all $y \in \Sigma^{+}$. Since the set of pre-images $\bigcup_{n \geq 0}\left\{x \in \Sigma^{+}: \sigma^{n} x=z\right\}$ of any point in $z \in \Sigma^{+}$ is dense, we see from this argument that $w$ is nowhere zero.

Now consider the function $W: \Sigma^{+} \times G \rightarrow \mathbb{C}^{m}$ defined by $W(x, g)=R_{\chi}\left(g^{-1}\right) w(x)$. Then

$$
\begin{aligned}
W(\sigma x, \kappa(x) g) & =R_{\chi}\left(g^{-1}\right) R_{\chi}\left(\kappa(x)^{-1}\right) w(\sigma x) \\
& =R_{\chi}\left(g^{-1}\right) e^{i v(y)-i a} w(x) \\
& =e^{i v(y)-i a} W(x, g) .
\end{aligned}
$$

Iterating this argument, we have that, for each $x \in \Sigma^{+},\left\{W\left(\sigma^{n} x, \kappa_{n}(x)\right): n \geq 0\right\}$ is contained in the compact set $\left\{e^{i c} W(x, 1): c \in \mathbb{R}\right\}$. By Lemma 5.1, $\widetilde{\sigma}: \Sigma^{+} \times G \rightarrow \Sigma^{+} \times G$ is transitive, and so we can choose $x \in \Sigma^{+}$such that $\left\{\left(\sigma^{n} x, \kappa_{n}(x)\right): n \geq 0\right\}$ is dense in $\Sigma^{+} \times G$. It follows that

$$
\left\{R_{\chi}\left(g^{-1}\right) w(x): g \in G\right\} \subset\left\{e^{i c} w(x): c \in \mathbb{R}\right\},
$$

so the representation leaves invariant a 1-dimensional subspace, contradicting $m>1$.

Combining Lemma 5.2 with the arguments in Chapter 4 of [12], which show how the transfer operator determines the analytic behaviour of $L_{\sigma}(s, t, \chi)$. We have the following.

Proposition 5.3 Let $R_{\chi}$ be an $m$-dimensional irreducible representation of $G$.

(i) If $m>1$ the $L_{\sigma}(s, t, \chi)$, and hence $L(s, t, \chi)$, is non-zero and analytic in a neighbourhood of $\left\{s: \operatorname{Re}(s) \geq h^{*}\right\} \times \mathbb{T}^{b}$.

(ii) If $m=1$ then $L_{\sigma}(s, t, \chi)$, and hence $L(s, t, \chi)$, is non-zero and analytic in a neighbourhood of $\left(h^{*}+i \tau, t\right)$ unless $\mathcal{L}_{-\left(h^{*}+i \tau\right) r+\langle\xi+2 \pi i t, f\rangle, \chi \circ \kappa}$ has 1 as an eigenvalue.

We end the section by noting that if $\mathcal{L}_{-}\left(h^{*}+i \tau\right) r+\langle\xi+2 \pi i t, f\rangle, \chi \circ \kappa$ has 1 as an eigenvalue then

$$
e^{-i \tau r^{n}(x)+2 \pi i\left\langle t, f^{n}(x)\right\rangle} \chi\left(\kappa_{n}(x)\right)=1
$$

whenever $\sigma^{n} x=x$.

\section{Equidistribution results}

In this section we will prove Theorems $1.2,1.3$ and 1.4. We will work with higher order logarithmic derivatives of the $L$-functions. Write $v=[b / 2]$ and define

$$
\begin{aligned}
\eta(s, t, \chi) & :=\frac{\partial^{\nu+1}}{\partial s^{\nu+1}} \log L(s, t, \chi) \\
& =\sum_{n=1}^{\infty} \sum_{\gamma \in \mathcal{P}(\phi)} n^{\nu}(-l(\gamma))^{\nu+1} \chi\left(\mathfrak{h}(\gamma)^{n}\right) e^{(-s l(\gamma)+2 \pi i\langle t,[\gamma]\rangle+\langle\xi,[\gamma]\rangle) n} .
\end{aligned}
$$

It will now be convenient to introduce a set $\mathcal{Q}(\phi, \alpha)$ consisting of all, not necessarily prime, $\phi$-periodic orbits with homology class $\alpha$. For $\alpha \in H_{1}(M, \mathbb{Z})$, we also define

$$
\eta_{\alpha}(s, \chi)=\int_{\mathbb{T}^{b}} e^{-2 \pi i\langle t, \alpha\rangle} e^{-\langle\xi, \alpha\rangle} \eta(s, t, \chi) d t
$$




$$
=\sum_{n=1}^{\infty} \sum_{\gamma^{n} \in \mathcal{Q}(\phi, \alpha)} n^{\nu}(-l(\gamma))^{\nu+1} \chi\left(\mathfrak{h}(\gamma)^{n}\right) e^{-s n l(\gamma)},
$$

where $\gamma^{n}$ is the periodic obtained by traversing the prime periodic orbit $\gamma n$ times.

We will now prove our equidistribution results. By Proposition 2.1, it is enough to work with a class function of the form

$$
F=\sum_{i=0}^{n} a_{i} \chi_{i}
$$

where the $\chi_{i}$ are characters of irreducible unitary representations, with $\chi_{0}=\mathbb{1}$ and $a_{0}=$ $\int F d$ Haar. We define

$$
\eta_{\alpha}(s, F)=\sum_{i=0}^{n} a_{i} \eta_{\alpha}\left(s, \chi_{i}\right)=\sum_{n=1}^{\infty} \sum_{\gamma^{n} \in \mathcal{Q}(\phi, \alpha)} n^{\nu}(-l(\gamma))^{\nu+1} F\left(\mathfrak{h}(\gamma)^{n}\right) e^{-s n l(\gamma)} .
$$

\subsection{Case 1: $G$ semisimple}

In this case, $G$ has no non-trivial one-dimensional representations. When $R_{\chi}$ in a $m$ dimensional representation with $m>1$, part (i) of Proposition 5.3 gives that $\eta(s, t, \chi)$ is non-zero and analytic of a neighbourhood of $\left\{s: \operatorname{Re}(s) \geq h^{*}\right\} \times \mathbb{T}^{b}$ and hence $\eta_{\alpha}(s, \chi)$ is analytic in a neighbourhood of $\left\{s: \operatorname{Re}(s) \geq h^{*}\right\}$. Furthermore, the analysis of [17] shows that $\eta_{\alpha}(s, \mathbb{1})$ is analytic in a neighbourhood of $\left\{s: \operatorname{Re}(s) \geq h^{*}\right\} \backslash\left\{h^{*}\right\}$. We also have the following result, which follows immediately from Lemma 3 of [17].

Lemma 6.1 There exists $C>0$ (independent of $\alpha$ and $F$ ) such that the following hold.

(i) If $b$ is even then

$$
\lim _{\varsigma \rightarrow h^{*}}\left(\eta_{\alpha}(\varsigma+i \tau, F)-\frac{(-1)^{\nu+1} C a_{0}}{\varsigma+i \tau-h^{*}}\right)
$$

exists for almost every $\tau \in \mathbb{R}$ and is locally integrable. Moreover, the difference is dominated by a locally integrable function for $\varsigma>h^{*}$.

(ii) If $b$ is odd then

$$
\lim _{\varsigma \rightarrow h^{*}}\left(\eta_{\alpha}(\varsigma+i \tau, F)-\frac{(-1)^{\nu+1} C \pi^{1 / 2} a_{0}}{\left(\varsigma+i \tau-h^{*}\right)^{1 / 2}}\right)
$$

exists for almost every $\tau \in \mathbb{R}$ and is in the Sobolev space $W_{\mathrm{loc}}^{1,1}(\mathbb{R})$. Moreover, the difference is dominated by a locally integrable function for $\varsigma>h^{*}$.

When $b$ is even we can use the Wiener-Ikehara Tauberian theorem and when $b$ is odd we can use a version of the Agmon-Delange Tauberian theorem from [7] to deduce that

$$
\sum_{n=1}^{\infty} \sum_{\gamma^{n} \in \mathcal{Q}_{T}(\phi, \alpha)} n^{\nu} l(\gamma)^{1+b / 2} F\left(\mathfrak{h}(\gamma)^{n}\right) \sim C e^{-\langle\xi, \alpha\rangle}\left(\int F d \text { Haar }\right) e^{h^{*} T},
$$

as $T \rightarrow \infty$, where $C>0$ is independent of $\alpha$. It easily follows, since non-prime periodic orbits have a slower exponential growth rate that

$$
\sum_{\gamma \in \mathcal{P}_{T}(\phi, \alpha)} l(\gamma)^{1+b / 2} F(\mathfrak{h}(\gamma)) \sim C e^{-\langle\xi, \alpha\rangle}\left(\int F d \text { Haar }\right) e^{h^{*} T},
$$


as $T \rightarrow \infty$. Decomposing $F$ into real and imaginary parts, and then these into positive and negative parts, we can use standard partial summation techniques to conclude that

$$
\sum_{\gamma \in \mathcal{P}_{T}(\phi, \alpha)} F(\mathfrak{h}(\gamma)) \sim C e^{-\langle\xi, \alpha\rangle}\left(\int F d \text { Haar }\right) \frac{e^{h^{*} T}}{T^{1+b / 2}},
$$

as $T \rightarrow \infty$. Hence Theorem 1.2 holds by comparing this with the asymptotic for $\mathcal{P}_{T}(\phi, \alpha)$, i.e. the case $F=1$.

\subsection{Case 2: $G$ not semisimple}

If $G$ is not semisimple then it has non-trivial one-dimensional representations (characters). Let $\chi: G \rightarrow \mathbb{T}^{1}$ be such a non-trivial character. We need to show that, under the hypotheses of Theorem 1.3, $L(s, t, \chi)$ is non-zero and analytic on a neighbourhood of $\left\{s: s \geq h^{*}\right\} \times \mathbb{T}^{b}$. If $\operatorname{Re}(s)>h^{*}$ then we automatically have convergence to a non-zero analytic function, so we only need to avoid a singularity at $\left(h^{*}+i \tau, t\right)$ for any $\tau \in \mathbb{R}$ and $t \in \mathbb{T}^{b}$. This amounts to showing that $\mathcal{L}_{-\left(h^{*}+i \tau\right) r+\langle\xi+2 \pi i t, f\rangle, \chi}$ does not have 1 as an eigenvalue. In view of the comment after Proposition 5.3, it is enough to show that

$$
e^{-i \tau l(\gamma)+2 \pi i\langle t,[\gamma]\rangle} \chi(\mathfrak{h}(\gamma))=1
$$

cannot hold for all $\gamma \in \mathcal{P}(\phi)$. If it were to hold then, in particular, considering null homologous periodic orbits, we would have

$$
e^{-i \tau l(\gamma)} \chi(\mathfrak{h}(\gamma))=1
$$

for all $\gamma \in \mathcal{P}(\phi, 0)$. Applying hypothesis (iii) of Theorem 1.3, this implies that $\tau=0$ and $\chi=\mathbb{1}$, a contradiction. The proof now continues as in the semisimple case.

To end the section, we prove Theorem 1.4. Consider again the equation

$$
e^{-i \tau l(\gamma)+2 \pi i\langle t,[\gamma]\rangle} \chi(\mathfrak{h}(\gamma))=1 \quad \forall \gamma \in \mathcal{P}(\phi),
$$

where $\chi: G \rightarrow \mathbb{T}^{1}$ is a non-trivial character. If $\phi_{t}$ has time-reversal symmetry, for each $\gamma \in \mathcal{P}(\phi)$, we can consider its time-reversed twin $\iota(\gamma)$, which satisfies $l(\iota(\gamma))=l(\gamma)$, $[\iota(\gamma)]=-[\gamma]$ and $\mathfrak{h}(\iota(\gamma))=\mathfrak{h}(\gamma)^{-1}$. Thus we obtain

$$
e^{-2 i \tau l(\gamma)}=1 \quad \forall \gamma \in \mathcal{P}(\phi) .
$$

Since $\phi_{t}$ is weak-mixing, this can only hold when $\tau=0$. Substituting back, we get

$$
e^{2 \pi i\langle t,[\gamma]\rangle} \chi(\mathfrak{h}(\gamma))=1 \quad \forall \gamma \in \mathcal{P}(\phi)
$$

and then, restricting to null homologous periodic orbits,

$$
\chi(\mathfrak{h}(\gamma))=1 \quad \forall \gamma \in \mathcal{P}(\phi, 0) .
$$

Now the hypothesis of Theorem 1.4 gives $\chi=\mathbb{1}$, a contradiction as before. The proof then continues in the same way as above.

\section{Frame flows}

Let $V$ be a compact $d$-dimensional Riemannian manifold with negative sectional curvatures. Let $M=S V$ be the unit-tangent bundle and let $\phi_{t}: M \rightarrow M$ be the geodesic flow; this is a homologically full transitive Anosov flow. Let $N$ be the bundle of $d$-dimensional frames 
over $V$ and let $\psi_{t}: N \rightarrow N$ be the frame flow: $\psi_{t}$ parallel transports $\left(e_{1}, \ldots, e_{d}\right) \in N$ along the geodesic determined by $e_{1}$. Then the map $\left(e_{1}, \ldots, e_{d}\right) \mapsto e_{1}$ makes $N$ into a principal $\mathrm{SO}(d-1)$-bundle over $M$ so that $\psi_{t}$ factors to $\phi_{t}$.

The frame flow $\psi_{t}$ leaves invariant a measure which is locally the product of the (normalised) Riemannian volume on $M$ and the Haar measure on $\mathrm{SO}(d-1)$. When we discuss ergodicity of $\psi_{t}$ it will be with respect to this measure.

We note that if $d \geq 4$ then $\mathrm{SO}(d-1)$ is semisimple. (In fact, it is simple when $d$ is even.)

Theorem 7.1 Let $V$ be a compact negatively curved Riemannian manifold of dimension $d \geq 4$ such that the frame flow is ergodic. Then $\phi_{t}$ has homology-equidistributed holonomy.

Proof If $\psi_{t}: N \rightarrow N$ is ergodic then it is transitive. The other hypotheses of Theorem 1.2 are automatically satisfied and so the result holds.

Remark 7.2 If $V$ is a compact manifold that admits $C^{3}$ metrics of negative curvature then those metrics with ergodic frame flow form an open and dense set [2]. Moreover, if $V$ has a Riemannian metric with sectional curvatures in $\left[-c_{2},-c_{1}\right]$ for $0<c_{1}<c_{2}$. then the frame flow is known to be ergodic under the following circumstances:

(1) if $V$ has constant negative curvature [3];

(2) if $d$ is odd and $d \neq 7$ [4];

(3) if $d$ is even, $d \neq 8$ and $c_{1} / c_{2}>0.93$ [5];

(4) if $d=7$ or 8 and $c_{1} / c_{2}>0.99023 \ldots$ [6].

However, if $V$ is a compact negatively curved Kähler manifold with $d \geq 4$ (e.g. a quotient of complex hyperbolic space) then the frame flow is not ergodic due to the presence of an invariant complex structure. Examples are given by quotients of complex hyperbolic space or other rank 1 locally symmteric spaces of non-constant curvature. In these settings, homologyequidistribution of holonomy with respect to the appropriate (smaller) holonomy group was proved in [8] (see also [10]).

If $d=3$ then the frame bundle is a principle $G$-bundle over $S V$ with $G=\operatorname{SO}(2) \cong \mathbb{T}^{1}$. Since the frame flow is ergodic $d=3$ and the geodesic flow has time-reversal symmetry, we have the following corollary of Theorem 1.4.

Theorem 7.3 Let $V$ be a compact negatively curved Riemannian manifold of dimension 3 such that the frame flow is ergodic. Suppose that $\{\mathfrak{h}(\gamma): \gamma \in \mathcal{P}(\phi, 0)\}$ is not contained in a proper closed subgroup of $\mathrm{SO}(2)$. Then $\phi_{t}$ has homology-equidistributed holonomy.

Remark 7.4 (Convex co-compact quotients) Let $\phi_{t}: \Omega \rightarrow \Omega$ be a (weak-mixing) hyperbolic flow on a set $\Omega$ contained in a manifold $M$. We may still use the analysis of [17] to obtain an asymptotic counting result for periodic orbits in a prescribed homology class in $H_{1}(M, \mathbb{Z})$ provided we assume that every class in $H_{1}(M, \mathbb{Z})$ is represented by a periodic orbit. (We may also obtain results with respect to $H_{1}(\Omega, \mathbb{Z})$ provided this is assumed to have finite rank.) In particular, this holds for the geodesic flow over a convex co-compact quotient of a pinched negative curvature complete simply connected manifold, where $\Omega$ is the non-wandering set. Thus we may obtain similar equidistribution results from the frame flow in this setting.

Acknowledgements The author is grateful to Mark Pollicott for bringing the paper [10] to his attention.

Open Access This article is distributed under the terms of the Creative Commons Attribution 4.0 International License (http://creativecommons.org/licenses/by/4.0/), which permits unrestricted use, distribution, and reproduction in any medium, provided you give appropriate credit to the original author(s) and the source, provide a link to the Creative Commons license, and indicate if changes were made. 


\section{References}

1. Bowen, R.: Symbolic dynamics for hyperbolic flows. Am. J. Math. 95, 429-460 (1973)

2. Brin, M.: Topological transitivity of one class of dynamical systems and flows of frames on manifolds of negative curvature. Funktsional. Anal. i Prilozhen. 9, 9-19 (1975). [English translation: Funct. Anal. Appl. 9, 8-16, (1975)]

3. Brin, M.: The topology of group extensions of dynamical systems. Mat. Zam. 18, $453-465$ (1976). (English translation: Math. Notes 18, 858-864)

4. Brin, M., Gromov, M.: On the ergodicity of frame flows. Invent. Math. 60, 1-7 (1980)

5. Brin, M., Karcher, H.: Frame flows on manifolds with pinched negative curvature. Compos. Math. 52, 275-297 (1984)

6. Burns, K., Pollicott, M.: Stable ergodicity and frame flows. Geom. Ded. 98, 189-210 (2003)

7. Katsuda, A., Sunada, T.: Closed orbits in homology classes. Inst. Hautes Études Sci. Publ. Math. 71, 5-32 (1990)

8. Kimoto, K., Wakayama, M.: Equidistribution of holonomy restricted to a homology class about closed geodesics. Forum Math. 14, 383-403 (2002)

9. Margulis, G., Mohammadi, A., Oh, H.: Closed geodesics and holonomies for Kleinian manifolds. Geom. Funct. Anal. 24, 1608-1638 (2014)

10. Oh, H., Pan, W.: Local mixing of frame flow and equidistribution for abelian covers (2016) (preprint)

11. Parry, W., Pollicott, M.: The Chebotarov theorem for Galois coverings of Axiom A flows. Ergod. Theory Dyn. Syst. 6, 133-148 (1986)

12. Parry, W., Pollicott, M.: Zeta functions and the periodic orbit structure of hyperbolic dynamics. Astérisque 187-188, 1-268 (1990)

13. Plante, J.: Anosov flows. Am. J. Math. 94, 729-754 (1972)

14. Pollicott, M., Sharp, R.: Asymptotic expansions for closed closed orbits in homology classes. Geom. Ded. 87, 123-160 (2001)

15. Price, J.: Lie Groups and Compact Groups. London Mathematical Society Lecture Note Series, vol. 25. Cambridge University Press, Cambridge (1977)

16. Sarnak, P., Wakayama, M.: Equidistribution of holonomies about closed geodesics. Duke Math. J. 100, 1-57 (1999)

17. Sharp, R.: Closed orbits in homology classes for Anosov flows. Ergod. Theory, Dyn. Syst. 13, 387-408 (1993) 